\title{
Unbearable Pruritus After Withdrawal of (Levo)cetirizine
}

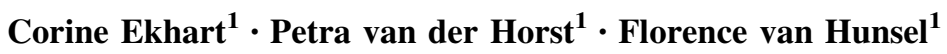

Published online: 26 November 2016

(C) The Author(s) 2016. This article is published with open access at Springerlink.com

\begin{abstract}
Twelve cases of unbearable pruritus several days after withdrawal of (levo)cetirizine were reported to the Netherlands Pharmacovigilance Centre Lareb. Eleven reports concerned women and one report concerned a man, aged 19-58 years. These patients had been using these antihistamines continuously for months or years. They had tried to stop using antihistamines on several occasions but felt unable to withdraw the drug because of the unbearable maddening itch. Finally, slowly tapering the drug or using a short course of corticosteroids helped to withdraw (levo)cetirizine. The Naranjo assessment score ranged from two to four for all the cases, indicating a possible relationship.
\end{abstract}

\section{Key Points}

(Levo)cetirizine is widely used in the treatment of allergic rhinitis and is tolerated well.

However, generalised pruritus after withdrawal can occur, making patients feel unable to stop using the antihistamine.

Gradually reducing the dose of the (levo)cetirizine or a short course of corticosteroids might help in withdrawing the antihistamine.

Corine Ekhart

c.ekhart@lareb.nl

1 Netherlands Pharmacovigilance Centre Lareb, Goudsbloemvallei 7, $5237 \mathrm{MH}$ 's Hertogenbosch, The Netherlands

\section{Introduction}

Allergic rhinitis is an inflammatory immunoglobulin E-mediated disease with symptoms of sneezing, nasal pruritus, nasal congestion and nasal discharge. Triggering allergens include seasonal pollens and moulds, as well as dust mites and pets. Allergic rhinitis can be seasonal or year round, with symptoms being intermittent or persistent [1]. Sensitisation to inhaled allergens begins during the first year of life. The prevalence of allergic rhinitis peaks in the second to fourth decades of life and then gradually diminishes [2]. Allergic rhinitis is a common disease, with a prevalence estimated between 10 and $30 \%$ of the population [3]. Treatment options include $\mathrm{H}_{1}$-antihistamines, intranasal glucocorticoids, leukotriene-receptor antagonists and allergen immunotherapy [2]. Oral antihistamines have been in use since 1940. These agents are effective for the relief of symptoms including rhinorrhoea, sneezing, itching, nasal blockage as well as associated ocular complaints. Oral antihistamines can be broadly categorised as first- or second-generation antihistamines. The first-generation antihistamines have side effects such as sedation and mucosal dryness, which limit their use. Second-generation antihistamines are highly selective for the $\mathrm{H}_{1}$ receptor and have limited penetration of the central nervous system. Cetirizine and levocetirizine belong to the second-generation antihistamines. Levocetirizine is the active R-enantiomer of cetirizine. The advantages of treatment with oral antihistamines include a rapid onset of action, once-daily dosing, maintenance of effectiveness with regular use and the availability of some drugs without a prescription. Maximum benefit is seen with continuous use, but in patients with intermittent symptoms use on an as-needed basis can provide significant symptom relief [1]. 
In the period from October 1989 to June 2016, the Netherlands Pharmacovigilance Centre Lareb received 12 reports of unbearable pruritus after withdrawal of cetirizine or levocetirizine. Four reports were reported by healthcare professionals and eight reports were reported by consumers. Striking in these reports is the occurrence of a maddening generalised itch after withdrawal of the antihistamine, which is reported to be different to the complaints that the antihistamines were used for in the first place.

\section{Case Reports}

Lareb received 12 reports of generalised pruritus after withdrawal of (levo)cetirizine (Table 1). The patients described pruritus without any other symptoms such as rashes or urticaria. The reports concerned 11 women and one man with a median age of 39 years (range 19-58 years). The pruritus occurred 1-3 days after withdrawal of long-term use of (levo)cetirizine. The patients had been using (levo)cetirizine continuously for months or years. Nine patients reported that they tried to stop using (levo)cetirizine several times but were not able to withdraw the drug because of the unbearable itch. One patient reported that a gradual reduction in dose helped and that after several weeks, the itching slowly receded and eventually disappeared. Another patient used a short course of corticosteroids. Five patients reported the use of concomitant medication. The concomitant medication was started years before the onset of the pruritus (1-13 years). The following case report (case 11 in Table 1), with a report date of 24 February, 2016, is representative of the cases reported to Lareb.

A 20-year-old woman had been using levocetirizine for hay fever for 2 years. Twenty-six hours after withdrawal she experienced pruritus. It started as a burning itch across the hands. The pruritus aggravated gradually across her head and was generalised after 2 days. It was unbearable, she could not sleep and was unable to go to work. In the past, she had tried to stop using levocetirizine several times, but every time there was more than $24 \mathrm{~h}$ between the intake of tablets, she would experience pruritus. The longer the period between the intake between two tablets, the worse the pruritus became. When she took levocetirizine, the pruritus would disappear within $30 \mathrm{~min}$. Therefore, she felt unable to withdraw levocetirizine. Ultimately, she was treated with prednisolone for 1 week and is recovering 3 weeks after withdrawal. The Naranjo causality score for this case was three.

\section{Discussion}

Pruritus is a common symptom that is experienced by all humans at some point in life. It occurs in a diverse range of skin diseases and may affect the skin, mucous membranes and cornea. The frequent occurrence of pruritus is supported by the results of epidemiological studies that found the prevalence of pruritus in the general population was approximately $8 \%$ [4].

Unbearable itch after withdrawal of antihistamines is not a common phenomenon. A PubMed search revealed no published reports of this association. However, there has been extensive discussion about the occurrence of pruritus after withdrawal of (levo)cetirizine on several patient forums and blogs $[5,6]$.

Antihistamines are used for the treatment of pruritus and therefore one would expect that withdrawal of antihistamines leads to the same symptoms for what the antihistamines were described. However, the patients in the cases reported to the Netherlands Pharmacovigilance Centre Lareb describe the generalised unbearable itch as a completely different itch for which (levo)cetirizine was described. In the majority of cases, the indication for use was hay fever.

Many internal diseases may present with itching symptoms. Pruritus is a frequent manifestation in patients with liver diseases and intra- or post-hepatic cholestasis, a sign of chronic renal insufficiency and a number of haematological diseases such as polycythaemia vera [7]. There is no indication in the reported cases that patients experienced any of these underlying diseases because there are no other reactions reported besides pruritus and no concomitant medication that would suggest the presence of such illnesses. Unfortunately, no laboratory values were provided in the reports to exclude underlying diseases, but there were four reports that were medically confirmed and these healthcare providers did not mention any other causes for the pruritus.

Although (levo)cetirizine has been reported to cause nephritis or hepatotoxicity $[8,9]$, this is an unlikely cause of the pruritus in these cases as the reaction only started after withdrawal of the drug and there were a number of patients with 'positive re-challenges'. Pruritus can be a side effect of a variety of drugs, such as morphine, opioids, angiotensin converting enzyme inhibitors, analgesics, vitamin A, contrast media, gold, chloroquine and sulphonamides [10]. However, all the concomitant medications that were reported had already been used years prior to the reaction and the time relation strongly links the pruritus to the withdrawal of (levo)cetirizine.

The Naranjo causality score ranged from two to four for all the cases. This is a relatively low score. However, the 


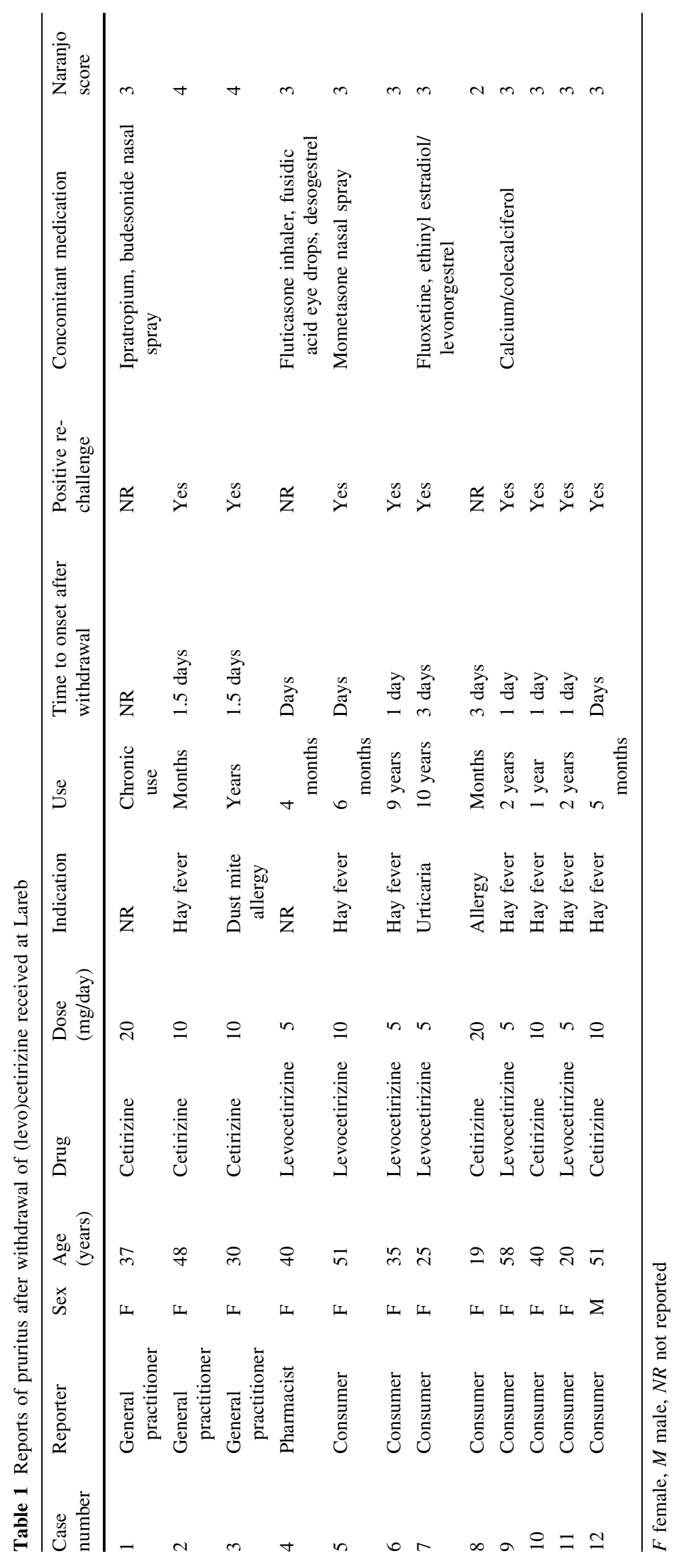


Naranjo causality score is not really suited for withdrawal reactions [11].

How long-term treatment with (levo)cetirizine may cause this withdrawal reaction is not known. $\mathrm{H}_{1}$ receptors mediate allergy in peripheral tissues and histaminergic neurotransmission in the central nervous system. The $\mathrm{H}_{1}$ receptor (H1R) gene is upregulated in patients with pollinosis. Its expression level is highly correlated with allergic symptom severity. Antihistamines are widely used as allergy treatments because they inhibit histamine signalling by blocking H1R or suppressing H1R signalling as inverse agonists [12]. Long-term treatment with antihistamines can decrease H1R gene expression to the basal level [13]. It is hypothesised that after stopping long-term treatment, H1R blockade disappears relatively fast (the half-life of (levo)cetirizine is 6-10 h). In the presence of histamine, this can lead to occupancy of the H1R and because the number of H1R was down-regulated owing to long-term treatment, a relatively high percentage of H1R will be occupied with histamine, possibly resulting in itch. Shortterm use will probably not lead to these symptoms because down-regulation of H1R has not been achieved in that time. It is remarkable that the patients reported at Lareb only experienced itch and no other symptoms induced by occupancy of the $\mathrm{H}_{1}$ receptor.

(Levo)cetirizine is a commonly prescribed drug in the Netherlands. Around 430,000 patients each year are prescribed (levo)cetirizine [14]. Levocetirizine and cetirizine are the only antihistamines in the Lareb database of which there are reports about this withdrawal reaction. There are no reports of unbearable itch after withdrawal of other antihistamines.

\section{Conclusion}

Based on the cases reported to the Netherlands Pharmacovigilance Centre Lareb, we suggest a causal relation between withdrawal of (levo)cetirizine and the occurrence of unbearable pruritus. The Naranjo assessment score ranged from two to four for all the cases, indicating a possible relationship. Healthcare professionals should be aware that in some patients this reaction can occur. Gradually reducing the dose of the (levo)cetirizine or a short course of corticosteroids might help in withdrawing the antihistamine.

\section{Compliance with Ethical Standards}

Funding No financial support was received for the conduct of this study or preparation of this article.

Conflict of interest Corine Ekhart, Petra van der Horst and Florence van Hunsel declare that they have no conflicts of interest.
Consent for publication Written informed consent was obtained from the patient in the detailed case description (case 11 in Table 1) for the publication of the case report. A copy of the consent may be requested for review from the corresponding author.

Open Access This article is distributed under the terms of the Creative Commons Attribution-NonCommercial 4.0 International License (http://creativecommons.org/licenses/by-nc/4.0/), which permits any noncommercial use, distribution, and reproduction in any medium, provided you give appropriate credit to the original author(s) and the source, provide a link to the Creative Commons license, and indicate if changes were made.

\section{References}

1. Seidman MD, Gurgel RK, Lin SY, et al. Clinical practice guideline: allergic rhinitis. Otolaryngol Head Neck Surg. 2015;152(1 Suppl.):S1-43.

2. Wheatley LM, Togias A. Clinical practice: allergic rhinitis. N Engl J Med. 2015;372(5):456-63.

3. Wang XY, Lim-Jurado M, Prepageran N, et al. Treatment of allergic rhinitis and urticaria: a review of the newest antihistamine drug bilastine. Ther Clin Risk Manag. 2016;12:585-97.

4. Dalgard F, Svensson A, Holm JO, Sundby J. Self-reported skin morbidity among adults: associations with quality of life and general health in a Norwegian survey. J Investig Dermatol Symp Proc. 2004;9(2):120-5.

5. The People's Pharmacy. Cetirizine (Zyrtec) withdrawal and unbearable itching. Available from: http://www. peoplespharmacy.com/2013/05/06/cetirizine-zyrtec-withdrawalunbearable-itching/. Accessed 21 Nov 2016.

6. MedHelp. Addicted to Zyrtec? Available from: http://www. medhelp.org/posts/Allergy/addicted-to-zyrtec/show/600862. Accessed 21 Nov 2016.

7. Peharda V, Gruber F, Kaštelan M, et al. Pruritus an important symptom of internal diseases. Acta dermatovenerologica Alpina Panonica et Adriatica. 2000;9(3):92-104.

8. Raghavendran R, Shipman AR, Langman G, et al. Acute interstitial nephritis secondary to long-term use of cetirizine for the treatment of urticaria pigmentosa. Clin Exp Dermatol. 2013;38(1):100-1.

9. Ekiz F, Yüksel I, Ekiz O, et al. Levocetirizine induced hepatotoxicity in a patient with chronic urticaria. Ann Hepatol. 2011;10(2):237-8.

10. Yonova D. Pruritus in certain internal diseases. Hippokratia. 2007;11(2):67-71.

11. Naranjo CA, Busto U, Sellers EM, et al. A method for estimating the probability of adverse drug reactions. Clin Pharmacol Ther. 1981;30(2):239-45.

12. Kitamura $Y$, Nakagawa $H$, Fujii $T$, et al. Effects of antihistamine on up-regulation of histamine $\mathrm{H} 1$ receptor mRNA in the nasal mucosa of patients with pollinosis induced by controlled cedar pollen challenge in an environmental exposure unit. J Pharmacol Sci. 2015;129(3):183-7.

13. Mizuguchi H, Orimoto N, Kadota $\mathrm{T}$, et al. Suplatast tosilate alleviates nasal symptoms through the suppression of nuclear factor of activated T-cells-mediated IL-9 gene expression in toluene-2,4-diisocyanate-sensitized rats. $\mathrm{J}$ Pharmacol Sci. 2016;130(3):151-8.

14. GIP database. Drug information system of the Dutch Health Care Insurance Board. Available from: http://www.gipdatabank.nl. Accessed 21 Nov 2016. 